\title{
ANALGESIA PREEMPTIVA NA ODONTOLOGIA: UMA BREVE REVISÃO DE LITERATURA
}

Monnique Soares Pacheco MONTEIRO ${ }^{1}$ Aladim Gomes LAMEIRA ${ }^{2}$

\author{
${ }^{1}$ Acadêmica do Curso de Odontologia da Universidade Federal do Pará - UFPA. \\ monnique.futura@yahoo.com.br \\ ${ }^{2}$ Doutor em Patologia Bucal \\ Professor Associado I do Curso de Odontologia da Universidade Federal do Pará. \\ alagolameira@ufpa.br
}

Recebido em: 30/05/2014 - Aprovado em: 28/09/2014 - Disponibilizado em: 15/12/2014

\begin{abstract}
RESUMO
O presente trabalho tem por intuito realizar uma revisão de literatura à respeito da analgesia preemptiva, na qual é um tratamento farmacológico iniciado antes de um procedimento cirúrgico, minimizando suas manifestações clínicas, principalmente a prevenção da hiperalgesia pós-cirúrgica. Sendo este, um tema pouco esclarecido devido suas controvérsias e, de pouco conhecimento pelos profissionais da odontologia, tornando assim, de suma importância para viabilizar o conforto pós-operatório de muitos pacientes. Desta forma, objetivo identificar a eficácia desta técnica visando propor um protocolo de atendimento com os medicamentos disponíveis no mercado. Em conformidade com alguns estudos, os protocolos medicamentosos considerados mais eficazes são as associações entre corticóides e analgésicos de ação periférica por via oral, sendo variável o tempo de sua prescrição.
\end{abstract}

Palavras - chave: Analgesia. Pós-operatório. Hiperalgesia. Preemptiva. Controle da dor.

\begin{abstract}
The present work has as its achieve a review of the literature concerning the preemptive analgesia, in which is a pharmacological treatment started before a surgical procedure, minimizing its clinical manifestations, especially the prevention of postoperative hyperalgesia. This being, a little savvy theme due to its controversy and little knowledge by practitioners of dentistry, making thus of paramount importance to achieve postoperative comfort of many patients. In this way, aim to identify the effectiveness of this technique in order to propose a protocol of care with the medicines available in the market. According to some studies, considered the most effective drug protocols are associations between steroids and painkillers peripheral action orally, prescribing time variable.
\end{abstract}

Keywords: Analgesia. Postoperative. Hyperalgesia. Preemptive. Pain control.

\section{INTRODUÇÃO}

Dos muitos tratamentos odontológicos, a cirurgia é dos mais invasivos por isso a dor inflamatória se estabelece como um componente mais importante em resposta à injúria tecidual (PINHEIRO et al. 2004).
Segundo Malamed (2001) é mais fácil evitar a dor à reduzi-la depois de estabelecida.

O controle da dor e da inflamação pósoperatória, são preocupações dos cirurgiõesdentistas, bem como, o bem estar dos pacientes nos pós cirúrgico (ANDRADE, 2006). 
O termo "preemptiva" significa eliminar o problema antes que ele apareça. $\mathrm{O}$ primeiro conceito surgiu com Crile (1913) ele observou que o bloqueio da transmissão da dor antes da incisão cirúrgica, reduzia a mortalidade pósoperatória. A analgesia preemptiva é um assunto controverso e que tem despertado interesse de vários profissionais da saúde. sendo definida como um regime analgésico que tem inicio antes da nocicepção, objetivando prevenir a hiperalgesia e amplificação da dor (JOSHI et al., 2004; ONG et al., 2004; KACZMARZYK et al., 2010).

O pioneiro a usar o termo foi Wall (1988) com seu estudo sobre administração de analgésicos opióides e anestésicos locais de forma isolada ou associada antes de incisões cirúrgicas, observou uma diminuição da intensidade da dor pós-cirúrgica. Acredita-se que essa técnica proporciona conforto ao paciente e reduz o consumo de analgésico no pós-operatório, consequentemente na melhor recuperação do paciente.

Há muitas controvérsias sobre esse método devido ao regime farmacológico a ser adotado e o momento a ser empregado. Para Kissin (2000) essa prática reduz a intensidade e duração da dor pós- operatória e justifica o termo "preemptiva", ou seja, analgesia que precede o estímulo doloroso. $\mathrm{O}$ regime farmacológico deve ser introduzido antes do trauma cirúrgico, imediato após lesão tecidual e antes o inicio da dor (PINHEIRO et al.2004).
Para Kelly (2001) a analgesia preemptiva é uma terapia antinoceptiva, sendo recomendado para prevenção de sensibilizações centrais e periféricas, diminuindo ou modulando a sensação dolorosa.

Diante exposto, este trabalho tem por finalidade apresentar e discutir estudos encontrados na literatura referentes à analgesia preemptiva e sua aplicabilidade na odontologia.

\section{REVISÃO DE LITERATURA}

A dor é um fenômeno biológico, cuja expressão é subjetiva. Na odontologia existem alguns procedimentos invasivos, a exemplo as cirurgias, por isso a dor inflamatória está presente e, é a principal em resposta à injúria tecidual (PINHEIRO, 2004).

O termo "preemptivo" significa eliminar o problema antes que apareça. A sensibilização das fibras nociceptivas causa mudanças neurais e comportamentais e podem persistir após a cessão do estímulo.

No intuito de minimizar as manifestações clinicas de traumas cirúrgicos indicam-se fármacos com o objetivo de bloquear os mediadores químicos responsáveis pela resposta inflamatória (DIONE et al., 2003). Para Grape e Tramer (2007) a analgesia preemptiva tem três objetivos: diminuir a dor aguda provocada pelo trauma cirúrgico; 
prevenir o surgimento da dor referida e crônica; e prevenir a dor do pós- operatória.

Segundo Aida et al., 1999; Katz;Mccartney, 2002) a intensidade da dor pós-operatória depende: do tipo e duração da cirurgia, extensão e natureza da lesão tecidual, dos fármacos escolhidos e da analgesia pósoperatória.

Na década de 80, estudos em animais sugeriram que o bloqueio periférico preemptivo de estímulos dolorosos ao sistema nervoso central com anestesia geral (AG) e anestesia local (AL) e com o uso de analgésicos não esteroidais poderiam ser benéficos em atenuar a dor pós-operatória. Estudos clínicos sugerem combinar AG com AL, e talvez com o uso de AINES com ou sem narcóticos para reduzir a severidade da dor pós- operatória (KAUFMAN et al., 2005).

Além disso, Foreman (1995) corrobora quanto a associação da anestesia geral com o bloqueio regional e local. Concluiu que houve alterações cardiovasculares benéficas, bem como a diminuição do uso de medicamentos analgésicos no pós-cirúrgico.

Estudos realizados por Ong et al. (2004 b) investigaram o efeito do óxido nitroso em exodontias de terceiros molares mandibulares impactados bilateralmente tanto no pré quanto no pós- cirúrgico. Todos os pacientes de um lado receberam como pré- cirúrgico óxido nitroso a $50 \%$ por 20 min. e pós- operatório 20 min de oxigênio a $100 \%$ como placebo, no lado oposto receberam oxigênio a $100 \%$ durante $20 \mathrm{~min}$. no pré- cirúrgico e no póscirúrgico óxido nitroso a $50 \%$ por $20 \mathrm{~min}$. Eles concluíram que óxido nitroso não tem efeito analgésico quando administrado no précirúrgico.

Com o passar do tempo os estudos se estenderam as drogas analgésicas. Existem três grupos de drogas utilizadas no controle da dor pós-cirúrgica, no qual são comumente utilizadas na odontologia: anti-inflamatórios esteroidais (corticosteróides); não esteroidias (AINES) e os analgésicos de ação central e periférica (ROCHA et al., 2003). Estes têm efeitos geralmente atribuídos a supressão de mediadores químicos da resposta inflamatória no local da injúria (DIONE ,2003).

Outro fator que muitas vezes traz desconforto ao paciente é o edema e, sua diminuição reduz a dor e aumenta o limite de abertura bucal (PEDERSEN, 1985). O grupo de fármacos mais indicados para estas finalidades são os corticóides, existindo diversos estudos que comprovam sua eficácia, a exemplo disto, destaco o experimento realizado por Pedersen (1985) com utilização de $4 \mathrm{mg}$ de dexametasona, por via intramuscular (no masseter) e oral em 500 pacientes pré-cirúrgicos de exodontia de terceiros molares mandibulares incluso, constatando sua eficiência na prevenção do edema, trismo e dor pós-operatória. 
Já Micó-llorens et al., (2006) avaliaram e compararam o efeito do metilprednisolona em dose única de 40mg antes das exodontias de terceiros molares impactados e um grupo controle que não recebeu a medicação, no qual a cirurgia consistiu em realizar uma osteotomia e odontosecção. E obtiveram como resultado a eficácia do corticóide em oferecer um pós-operatório mais confortável.

Do mesmo modo, Kang et al., (2010) avaliaram a eficácia da prednisolona e do ibuprofeno em 450 pacientes com indicação de exodontias de terceiros molares mandibulares inclusos, eles foram divididos em três grupos: grupo I recebeu prednisolona $10 \mathrm{mg}$ por via oral $1 \mathrm{~h}$ antes da cirurgia e $200 \mathrm{mg}$ de ibuprofeno também por via oral uma hora antes do procedimento cirúrgico; grupo II recebeu $20 \mathrm{mg}$ de prednisolona e $200 \mathrm{mg}$ de ibuprofeno por via oral $1 \mathrm{~h}$ antes da cirurgia; e o grupo III recebeu somente $200 \mathrm{mg}$ de ibuprofeno por via oral $1 \mathrm{~h}$ antes da cirurgia. Todos os grupos receberam imediatamente após as cirurgia 200mg de ibuprofeno e também o receituário com a mesma droga por 3 dias de 8 em 8 horas. Os resultados mostraram que não houve diferença significativa entre os grupos em relação a dor, trismo e edema.

Também, Sotto-Maior et al., (2011) realizaram um estudo para comparar os efeitos dos corticóides e os AINES inibidores da COX-2 em relação a dor, edema e trismo.
Foram realizadas exodontias de terceiros molares mandibulares impactados em 50 pacientes. Na primeira cirurgia recebiam 120 $\mathrm{mg}$ de etoricoxibe por via oral $1 \mathrm{~h}$ antes do procedimento cirúrgico, no lado oposto recebiam $4 \mathrm{mg}$ de dexametasona por via oral 1h antes da cirurgia. Ambos os casos foram receitados paracetamol $750 \mathrm{mg}$ de $6 / 6 \mathrm{~h} \mathrm{em}$ caso de dor. Os resultados obtidos foram semelhantes em ambos casos, não havendo diferença na prevenção da dor, trismo e edema.

Segundo experimento realizado por Oliveira (2012), tendo como sujeitos 30 pacientes com indicações de exodontia de terceiros molares inferiores retidos, no qual de um lado receberam $8 \mathrm{mg}$ de dexametasona por via oral $1 \mathrm{~h}$ antes da cirurgia e placebo de $8 / 8 \mathrm{~h}$ por 2 dias. No contra lateral receberam cetorolaco $10 \mathrm{mg}$ por via oral $1 \mathrm{~h}$ antes da cirurgia. $\mathrm{O}$ resultado do experimento indica que não houve diferença significativa em relação a dor, mas em relação ao trismo e edema a dexametasona foi mais eficaz.

Os AINES são comumente prescritos pelos dentistas para o alivio da dor e controle do edema no pós- cirúrgico (MENEZES, 2010). O mais utilizado é o ibuprofeno, já mostrado em estudos acima e, sua eficácia tem sido avaliada, sendo considerado um fármaco analgésico padrão ouro (MORSE et al., 2006; MERRY et al., 2010). 
Ultimamente, os cirurgiões dentistas têm prescrito os COXIBES (inibidores seletivos da ciclooxigenase 2) acreditando que estes possuem maior potência analgésica que os AINES. Segundo Lane (1997) este grupo de medicamentos são efetivos no controle da dor, porém não apresentam conforto analgésico maior que os AINES disponíveis (LANE, 1997; TANG et al., 2002).

Igualmente, Morse (2006) concluiu que o ibuprofeno e o rofecoxib possuem a mesma eficácia preemptiva em cirurgias de exodontias de terceiros molares mandibulares..

Ao mesmo tempo, Young et al.,(2006) compararam a eficácia do valdecoxibe na redução do incomodo causado pelo fio ortodôntico na pré e pós colocação. Foram selecionados três grupos: (I) placebo; (II) os que receberam $40 \mathrm{mg}$ valdecoxibe $30 \mathrm{~min}$ antes da colocação do fio; e o grupo III receberam $40 \mathrm{mg}$ de valdecoxibe $2 \mathrm{~h}$ depois da colocação do fio. Observou-se que no grupo II não houve aumento significativo do incomodo. Em contrapartida os grupos I e III mostraram um aumento significativo após $6 \mathrm{~h}$ da cirurgia com pico nas primeiras $24 \mathrm{~h}$.

Já Arantes (2007) comparou a eficácia do efeito preemptivo do cetoprofeno e do parecoxibe, em 60 pacientes submetidos a exodontia de terceiros molares mandibulares inclusos. Os resultados mostraram que o parecoxibe, administrado antes da cirurgia, foi mais eficaz que o cetoprofeno no controle da dor no tempo de $4 \mathrm{~h}$ após as cirurgias e menos efetivo no decorrer das $24 \mathrm{~h}$ após a intervenção.

\section{CONSIDERAÇÕES FINAIS E DISCUSSÕES}

A analgesia preemptiva consiste em um tratamento antinoceptivo que previne o estabelecimento da alteração do processamento central, prevenindo a amplificação da dor nos pós- operatório (CRILE, 1913; WOOLF, 1983;KISSIN,1996; DAHL; KEHLET,1993; PINHEIRO,2004; ANDRADE,2006).

A importância da analgesia preemptiva na odontologia se faz presente, devido a preocupação destes profissionais em controlar a dor no pós-operatório, pois é um dos fatores determinantes da eficácia do tratamento. E consequentemente houve o aumento da busca de protocolos farmacêuticos que diminuam os transtornos causados pelas intervenções mais invasivas.

Assim sendo, o objetivo deste trabalho foi discutir estudos encontrados na literatura referentes a analgesia preemptiva e sua aplicabilidade na odontologia identificando, assim, a eficácia da técnica de analgesia preemptiva na dor pós- operatória. Tendo a intenção de contribuir para futuros trabalhos sobre o tema em questão, bem como contribuir para que no futuro venha se estabelecer um 
protocolo farmacológico universal, que atenda as necessidades dos cirurgiões- dentistas.

Sabemos que existem evidências que comprovam sua efetividade, porém há uma controvérsia de qual droga ou protocolo utilizar, mas de acordo com a literatura exposta nesta pesquisa observamos três correntes: (I) uso de fármacos no précirúrgico, (II) nos pós operatório e (III) pré, trans e pós- cirúrgico.

Segundo a literatura apresentada a prevenção da dor pós-cirúrgica ocorre através de dois mecanismos: no bloqueio efetivo do estímulo nocivo gerado durante e póscirúrgico, bem como no período inflamatório (analgesia preemptiva); em que é considerado mais eficaz no tratamento pré- operatório do que no pós- cirúrgico (KISSIN, 1996).

Para Amantea et al., (1999) administrar uma droga analgésica antes da cirurgia, não é suficiente para produzir um efeito preemptivo. Alguns autores sugerem que se deve utilizar a analgesia em diferentes intervalos de tempo para controlar o estimulo doloroso.

Vários estudos têm sidos realizados no intuito de encontrar uma droga ideal no conforto do pós- operatório. White e Reuben (2002) e Jung et al(2005) relatam que o uso oral e de maneira preemptiva dos AINES tem indicado alta capacidade em fornecer analgesia pós-operatória eficaz. De acordo com Andrade (2006) e Vane (1998) um dos fármacos utilizados na odontologia e, que previnem a sensibilização dos nociceptores por meio da inibição da cicloxigenase, são os AINES, e sua potência varia conforme sua meia- vida plasmática e com a dose empregada.

Porém ainda não foi definido qual utilizar e sua posologia. $\mathrm{O}$ primeiro a ser estudado foi o diclofenaco de sódio $100 \mathrm{mg}$, Zacharias et al. (1996) e Bridgman et al. (1996), informaram que empregado preemptivamente não possuía efeito eficaz, mas se administrado imediatamente após a cirurgia é mais efetivo.

Os coxibes, segundo Ong et al,. (2005), Young et al., (2006), Arantes (2007) possuem efetividade quando administrados preemptivamente. Contudo alguns estudos apontam que o uso deste grupo medicamentoso deve ser evitado, devido aos elevados riscos cardiovasculares, também o alto custo. Conforme assevera Andrade (2006) que os riscos cardiovasculares estão ligados à dose e ao tempo de uso dos coxibes.

Uma droga bastante utilizada na odontologia é o corticoide. Para Barron et al., (2004) a dexametasona é efetiva quando utilizada preemptivamente em exodontias de terceiros molares. Corroborando com esta ideia Andrade (2006) afirma que a dexametasona e outros corticóides, como a betametasona, agem inibindo a fosfolipase A2, consequentemente previnem a sensibilização dos nociceptores. 
Mesmo com a comprovação da eficácia do uso preemptivo dos analgésicos de ação periférica e corticóides na analgesia pósoperatória, ainda não se têm um consenso de qual droga e regime terapêutico a ser empregado.

Portanto, muitos fatores influenciam no estabelecimento da dor pós- cirúrgica, dentre eles: a ansiedade do paciente, o tipo e duração da cirurgia, o fármaco utilizado e o momento da administração (pré, trans e pós- operatório), além da extensão e natura da lesão tecidual.

\section{REFERÊNCIAS BIBLIOGRÁFICAS}

AIDA, S.; BABA, H.; YAMAKURA, T. The effectiveness of preemptive analgesia varies according to the type of surgery: a randomized, double-blind study. Anesth Analg, v.89, p.711-716, 1999.

\section{ALMEIDA, F. M.; ANDRADE, E. D. Estudo} clínico comparativo dos efeitos de duas preparações de betametasona, sobre o edema, trismo e dor, decorrentes da remoção de terceiros molares retidos. Revista de Odontologia da Universidade de São Paulo, São Paulo, v. 14, n.1, p.26-33, jan. / fev. 1990.

ALMEIDA, F. M. et al. Sugestão de um protocolo farmacológico para o controle da dor decorrente da exodontia de terceiros
Ainda não se têm um consenso de qual droga e regime farmacológico adotar, mas devido os resultados dos estudos considerados nesta revisão de literatura, temos assim, indícios significativos a respeito da tendência de associar analgésico de ação periférica com corticóides por via oral. Desta forma, as considerações apresentadas neste estudo apontam para a importância e continuidade de pesquisas sobre o referido tema. molares mandibulares inclusos. Revista Paulista de Odontologia; v. 22, n.1, p.10-16, 2000 .

AMANTEA, B. et al. Preemptive analgesia or balanced periemptive analgesia? Minerva Anestesiol. Jan-Feb.; v.65, n.1-2, p.19-37, 1999.

ANDRADE, E. D. Terapêutica medicamentosa em odontologia. 2. Ed.; Editora: Artes Médicas, 2006.

ARANTES, V. M. N. Analgesia preemptiva do cetoprofeno e do parecoxibe em cirurgia para remoção de terceiros molares inclusos. [S.l.; s.n.]. 2006. 
ARMITAGE, E.N. Postoperative

painprevention or relief? British Journal of

Anaesthesia, v.63, p.136-138, 1989.

\section{BARRON, R.P. et al : Effect of}

\section{dexamethasone and dipyrone on lingual}

and inferior alveolar nerve hypersensitivity

following third molar extraction:

preliminary report. J O Pain,v.18, n.1, p. 6268,2004 .

BRIDGMAN, J. B.; GILLGRASS, T. G.;

ZACHARIAS, M. The absence of any preemptive analgesic effect for nonsteroidal antiinflammatory drugs. British Journal of Oral and Maxillofac Surgery, v.34, n.5, Oct., p.428-431, 1996.

CRILE, G. W. The kinetic theory of shock and its prevention through anoci-association (shockless operation). Lancet, v.185, p.7-16, 1913.

DAHL, J. B.; KEHLET, H. The value of preemptive analgesia in the treatment of postoperative pain. Br J. Anaesth., v.70, p.434-9, 1993.

DIONE,R.A et al: Dexamethasone suppresses periapheral prostanoid levels without analgesia in clinical model of acute inflammation. J O and maxillofacial Sugery; v.61, n.9,p.997-1003,2003.
FOREMAN, P.A.: Preemptive analgesia: the prevention on neurogenous orofacial pain. Anesthesia Progress. V.42, n.2,p.3640,1995 .

GORDON, S. M. et al. The differential effects of bupivacaine and lidocaine on prostaglandin E2 release, cyclooxygenase gene expression and pain in a clinical pain model. Anesth Analg.; v.106, n.1, p.321-7, Jan., 2008.

GRAPE,S; TRAMER,M.R.: Do we need preemptive analgesia for the treatment of postoperative pain? Best pract res clin anesthesiol .v. 21, p. 51-63,2007.

JOSHI, A. et al.: A double- blind randomized controlled clinical trial of the effect of preoperative ibuprofen, diclofenac, paracetamol whit codeine and placebo tablets for relief of postoperative pain after removal of impacted third molars. Br j oral maxillofac surg. v. 42,p.299-306,2004.

JUNG, Y. S. et al. The effects on postoperative oral surgery pain by varying NSAID administration times: comparison on effect of preemptive analgesia. doi:10.1016/j.tripleo.2005.02.065. 
KACZMARZYK, T. et al.: Preemptive effect of ketoprofen on postoperative pain following third molar sugery. A prospective, randomized, double- blinded clinical trial.

International journal of oral and maxillofacial surgery. V. 39, n. 7, p. 647652. Marc. 2010.

KANG,S.H et al.: Effect of preoperative prednisolone on clinical postoperative symptoms after surgical extractions of mandibular third molars. Austral dent. J. v.55,n.4, p. 462-467,2010.

KATZ,J; MCCARTNEY, C. J. L. Current status of preemptive analgesia. Curr Opin Anaesthesiol, v.15, p.435- 441, 2002.

KAUFMAN, E. et al. Preemptive analgesia and local anesthesia as a supplement to general anesthesia. Anesth Prog. Spring, v.52, n.1, p.29-38, 2005.

KELLY, D. J.; AHMAD, M.; BRULL, S. J. Preemptive analgesia II: recent advances and current trends. Can J Anaesth. v.48, n.11, p.1091-101, Dec. 2001.

KISSIN,I: Preemptive analgesia- why its is not always obvious. Anesthesiology, v.84,p. 1015-1019,1996.
KISSIN,I. Preemptive analgesia.

Anesthesiology. V.93, p.1138-1143,2000.

LANE,N.E.: Pain management in osteoarthritis: The role of cox-2 inhibitors. Journal of rheumatology; . v. 24, n. 49, p. 20-24, 1997.

\section{MALAMED,S.F.: Manual de anestesia}

local. 4.ed.São Paulo: Guanabara Koogan, 2001.

MEHLISCH, D. R. The effi cacy of combination analgesic therapy in relieving dental pain. J. Am Dent Assoc, Chicago, v. 133, n. 7, p. 861-871, July 2002.

MENEZES,S.A.F; CURY,P.R. Efficacy of nimesulid versus meloxicam in the control of pain, swelling and trismus following extraction of impacted lower third molar. Int. j Oral maxillofac surg. v.39,p.580-584,2010.

MERRY, A.F et al: Combined acetaminophen and ibuprofen for pain relief after oral sugery in adults: a randomized controlled trial. $\mathbf{B r} \mathbf{~}$ Anesthesiol. V.104,p. 80-88, 2010.

MICÓ-LLORENS, J. M. et al. Effi cacy of methylprednisolone in controlling complications after impacted lower third molar surgical extraction. Eur J Clin 
Pharmacol.;v.62, n.9, p.693-8, Aug/Sep, 2006.

MORSE, Z; TUMP,A; KEVELHAM,E.: Ibuprofen as a preemptive analgesic is as effective as rofecoxib for mandibular third molar sugery. Odontology; V.94,P. 59-63, 2006

OLIVEIRA,J.G.P.: Comparação do efeito anti-inflamatório da dexametasona e do cetorolaco em exodontias de terceiros molares infeiores.2012. 107f.

dissertação(mestrado) universidade Federal de Mato Grosso do Sul, Campo Grande,2012.

ONG, K. S.; TAN, J. M. Preoperative intravenous tramadol versus ketorolac for preventing postoperative pain after third molar surgery. Int J Oral Maxillofac Surg.; v.33, n.3, p.274-8. Apr, 2004.

ONG, K. S. et al. Preoperative ketorolac has a preemptive effect for postoperative third molar surgical pain. Int J Oral Maxillofac Surg. v.33, n.8, p.771-6, Dec., 2004a.

ONG, K. S. et al. A prospective randomized crossover study of the preemptive analgesic effect of nitrous oxide in oral surgery. Oral Surg Oral Med Oral Pathol Oral Radiol Endod.; v.98, n.6, p.637-42, Dec., 2004b.
ONG, K. S. et al. The efficacy of preoperative versus postoperative rofecoxib for preventing acute postoperative dental pain: a prospective randomized crossover study using bilateral symmetrical oral surgery. Clin J Pain. v.21, n.6, p.536-42, Nov./Dec., 2005.

PEDERSEN, A.: Decadronphosphale in the relief of complaints after third molar sugery. International journal of oral sugery, Copenhagen,v.14,n.3, p.235-240, 1985.

PINHEIRO, M. L. P. et al. Analgesia preemptiva em odontologia. Braz J Oral Sci. v.3, n.10; Abstracts - II International Dental Meeting - UNICAMP and XI Dental Meeting of Piracicaba, 2004.

REUBEN, S. S.; BHOPATKAR, S.; MACIOLEK, $\mathrm{H}$. The preemptive analgesic effect of rofecoxib after ambulatory arthroscopic knee surgery. Anesth Analg., v.94, p.55-59, 2002.

ROCHA, R. G. et al. O controle da dor em odontologia através da terapêutica medicamentosa. In: CONCLAVE ODONTOLÓGICO INTERNACIONAL DE CAMPINAS, 15. Anais... n.104 - Mar/Abr., 2003.

SOTTO-MAIOR,B.S et al.: Corticosteroids or cyclooxygenase 2 selective inhibitor 
medication for the management of pain and swelling after third molar surgery. $\mathbf{J}$

craniofac surg. V.22,n.2, p. 759-762,2011.

VANE, J. R.; BOTTING, R. M. Mechanism of action of nonsteroidal anti-infl ammatory drugs. Am J Med 1998104 (3A): 2S-8S;

Discussion 21S-22S.

WALL, P. D. The prevention of postoperative pain. Pain, v.33, p.289-290, 1988.

WHITE, P. F. The role of non-opioid analgesic techniques in the management of pain after ambulatory surgery. Anesth Analg., v.94, p.577-585, 2002.

WOOLF, C. J. Evidence for a central component of post-injury pain hypersensitivity. Nature,v.306, p.686$688,1983$.

YOUNG-SOO, Jung. The effects on postoperative oral surgery pain by varying NSAID administration times: Comparison on /dor.htm>. Acesso em: 09 jan. 2014. effect of preemptive analgesia. Oral Surg Oral Med Oral Pathol Oral Radiol Endod., v.100, n.5, p.559-63, Nov., 2005. YOUNG, A. N. et al. Evaluation of preemptive valdecoxib therapy on initial archwire placement discomfort in adults. Angle Orthod., v.76, n.2, p.251-9, Mar. 2006.

TANG,J. et al. Effect of parecoxib, a novel intravenous cyclooxygenase type-2 inhibitor, on the postoperative opioid requirement and quality of pain control. Anesthesiology. V.96,p. 1305-1309,2002.

ZACHARIAS, M.; HUNTER, K. M.;

BAKER, A. B. Effectiveness of preoperative analgesics on postoperative dental pain: a study. Anesth Prog., v.43, n.3, p.92-6, Summer 1996. Disponível em: <www. dor. med. br/ dorclinica 